\title{
Prediction of hemodynamic reactivity by electroencephalographically derived Pain Rating Index in children during general anesthesia: a prospective observational study
}

\section{Lei Wu}

Shanghai Children's Medical Center

\section{Siyuan Wang}

3201 Hospital

\section{Yanting Wang}

Shanghai Children's Medical Center

\section{Kan Zhang}

Shanghai Children's Medical Center

Jie Bai

Shanghai Children's Medical Center

\section{Mazhong Zhang}

Shanghai Children's Medical Center

Jijian Zheng ( $\sim$ zhengijian626@sina.com )

Shanghai Childrens Medical Center Affiliated to Shanghai Jiaotong University School of Medicine https://orcid.org/0000-0003-1903-9557

Research article

Keywords: Pain Rating Index, Wavelet index, Hemodynamic reactivity, Pediatric patients

Posted Date: June 26th, 2019

DOI: https://doi.org/10.21203/rs.2.10696/v1

License: (c) (i) This work is licensed under a Creative Commons Attribution 4.0 International License. Read Full License 


\section{Abstract}

Background: Pain rating index (PRI) is a novel measure of nociception based on integrated electroencephalogram parameters during general anesthesia. Wavelet index (WLI) as a sedation index reflects the patient's sedation state. This study aims to evaluate the performance of PRI and WLI to predict hemodynamic reactivity after tracheal intubation and skin incision in pediatric patients. Methods: 134 children undergoing elective general or urinary surgery were analyzed. PRI, WLI, heart rate (HR), and mean blood pressure (MBP) were measured at predefined time-points during tracheal intubation and skin incision. Receiver-operating characteristic (ROC) curves were computed to evaluate the predictive performance of $\mathrm{PRI}$ and WLI to measure hemodynamic reactivity (increase by more than $20 \%$ of either MBP or HR) during general anesthesia. Results: In 134 patients, positive reactivity of HR and MBP was observed in 95 (70.9\%) and 61 (45.5\%) patients induced by endotracheal intubation and 19 (14.2\%) and $24(17.9 \%)$ patients induced by skin incision. Using either HR or MBP reactivity induced by tracheal intubation as a dichotomous variable, the areas under the curves (AUCs) [95\% CI] of PRI and WLI were $0.81[0.73-0.87]$ and $0.58[0.49-0.67]$ with the best cutoff values of 62 and 49 ; The AUCs [ $95 \% \mathrm{Cl}]$ of PRI and WLI were $0.82[0.75-0.88]$ and $0.61[0.52-0.69]$ after skin incision, the best cutoff values of PRI and WLI were 60 and 46, respectively. Conclusions: PRI can predict hemodynamic reactivity with the best cutoff values of 62 and 60 after tracheal intubation and skin incision in pediatric patients during general anesthesia while WLI failed in predicting hemodynamic changes. Trial registration: Chinese Clinical Trial Register (Unique identifier: ChiCTR1800015969, Date of registration: May 3, 2018). Key words: Pain Rating Index, Wavelet index, Hemodynamic reactivity, Pediatric patients

\section{Background}

The response to noxious stimulation during general anesthesia mainly depends on the balance between nociceptive and anti-nociceptive forces [1-3]. Nociception in anesthetized patients is typically demonstrated as sympathetic response to surgery and other noxious stimuli [4,5]. Excessive nociception not only affects the stability of hemodynamics, but may also exacerbate postoperative pain, disturb the balance of inflammation, and cause adverse outcomes [6, 7]. Analgesic administration is the key component of enhancing anti-nociceptive forces, which is mainly determined by the anesthesiologist's clinical experience [8]. However, it is very challenging to reach a appreciate analgesic level without the guide of an objective and effective assessment tool of anti-nociceptive state since errors in dosage may result in adverse side effects.[9]

Although clinical signs are usually used to indicate whether the analgesic level is sufficient or insufficient (such as increase of pupillary diameter, hemodynamic changes), these parameters cannot be used to predict the response to noxious stimulation since they are the results of noxious stimulation $[10,11]$. Furthermore, many confounding factors such as hypovolemia, $\beta$-blockers, anticholinergic drugs can also interfere with these clinical signs [12]. In recent years, several noninvasive monitoring tools based on skin conductance (SC), pupil diameter, heart rate variability (HRV), amplitude from photoplethymography (Surgical Pleth Index) or analysis of electroencephalogram (EEG) signals to estimate the antinociceptive 
state during general anesthesia has been used in adults, but less common in children, which might be due to age-dependent characteristics of EEG, ECG or lack of pediatric electrodes [11, 13-17].

A novel measure reflecting antinociceptive state under general anesthesia based on integrated EEG analysis was developed with pediatric mode by Beijing Easymonitor Technology Co., Ltd, China. The parameter is Pain Rating Index (PRI) ranging from 0 to 100, reflecting the tolerance of cerebral cortex and subcortex to pain stimulation according to the company's manual instruction. Theoretically, it can be used to predict the response to noxious stimulation instead of the result of noxious stimulation during general anesthesia. Whether PRI can be used to predict the response to noxious stimulation during general anesthesia remains unknown. Wavelet Index (WLI), a parameter of monitoring depth of sedation developed by the same company based on Wavelet analysis, is similar to bispectral index (BIS) in reflecting the patient's sedation state, and even better when the patients are under muscle relaxation or facial paralysis $[18,19]$. Therefore, our primary purpose was to examine whether PRI can be used to predict the hemodynamic reactivity induced by endotracheal intubation and skin incision in pediatric patients. Our secondary purpose was to investigate the role WLI in predicting the hemodynamic reactivity induced by endotracheal intubation and skin incision in pediatric patients.

\section{Methods}

This prospective observational clinical study was approved by the Institutional Review Board of Shanghai Children's Medical Center (reference number: SCMCRB-k2018050, May 20, 2018) and registered in the www.chictr.org.cn (ChiCTR1800015969, May 3, 2018). The study was performed between May 2018 and September 2018. Written informed consent was obtained from parents or legal guardians the day before data collection and surgery. All data acquisition took place in the operating room.

\section{Patients}

American Society of Anesthesiologists physical status (ASA) I or II, aged from 6 months to 12 years old pediatric patients scheduled for elective general surgery and urinary surgery under general anesthesia were included in this study.

Exclusion criteria: Patients with brain diseases (history of epilepsy, autism, cognitive dysfunction), autonomic nervous system disorders, liver and renal diseases, endocrinological diseases, cardiac arrhythmia, preoperative chronic pain, symptoms of upper respiratory infection, administration of anticholinergic drugs, vasopressors, vasodilators blockers, or ketamine before and during the experiment were excluded.

\section{Anesthetic technique}


For pediatric patients younger than 4 years old, $0.5 \mathrm{mg} / \mathrm{kg}$ midazolam PO 20-30 mins before entering the operating room, other patients did not receive any premedication. On arrival in the operation room, intravenous access and basic monitoring with an electrocardiogram, and pulse oximetry were established, and a crystalloid infusion was started following 4-2-1 principals [16]. Noninvasive blood pressure in the arm was recorded every 1 min during the experiment.

For uncooperative patients, $0.1-0.15 \mathrm{mg} / \mathrm{kg}$ midazolam IV was administered first, the pre-stimulation mean blood pressure (MBP), pre-stimulation values of heart rate (HR), WLI and PRI were measured and recorded. During the experiment, ketamine, atropine or vasoactive drugs were not allowed to be administered except when critical situations occurred. Anesthesia management was left to the discretion of the anesthesiologist in charge, with no extra restrictions imposed by the study except mentioned above. Generally, children received an intravenous induction with fentanyl (1.5-3.0 $\mu \mathrm{g} / \mathrm{kg})$ or sufentanil $(0.15-0.3 \mu \mathrm{g} / \mathrm{kg})$, propofol (2-4 mg/kg), and rocuronium $0.6 \mathrm{mg} / \mathrm{kg}$. Endotracheal intubation was performed by a skilled anesthesiologist who has used video laryngoscope at least 50 times. After successful intubation, anesthesia was maintained using propofol $4-8 \mathrm{mg} / \mathrm{kg} / \mathrm{h}$ and remifentanil $0.25-0.4$ $\mu \mathrm{g} / \mathrm{kg} / \mathrm{min}$, with or without $1.5-2.5 \%$ sevoflurane. Mechanical ventilation was initiated after tracheal intubation with a mixture of $50 \% \mathrm{O}_{2}$ and $50 \%$ air, Pressure controlled ventilation mode was used. Tidal volume was $8-10 \mathrm{ml} / \mathrm{kg}$, adjustment of inspiration pressure and ventilator frequency to keep end-tidal $\mathrm{CO}_{2}$ pressure between 35 and $45 \mathrm{mmHg}$.

\section{Study protocol and Analgesia Monitoring Device}

The anesthesiologist in charge was blind to the research protocol and HXD-I multi-function combination monitor (for WLI and PRI recording, Beijing Easymonitor Technology Co., Ltd., China) and responsible for determining anesthesia protocol and the timing of endotracheal intubation. The research team was responsible for recording PRI, WLI, HR, and MBP just before intubation and skin incision, and one minute after intubation and skin incision [20].

After the skin on forehead and mastoid was cleaned by water or alcohol cotton balls, EEG collection electrodes were placed $1-1.5 \mathrm{~cm}$ above center point between eyebrows in the forehead (FZ), above bilateral eyebrows (Left FP1, right FP2), and bilateral mastoid sites (left C1, right C2). Real-time EEG status and time trend of PRI and WLI data were displayed on the monitor screen in pediatric mode (Figure 1).

\section{Statistical Analysis}

Continuous data are presented as the mean $\pm \mathrm{SD}$, or median with interquartile range $[\mathrm{IQR}]$ according to the normality of the distribution, categorical variables as category counts and percentages. The continuous outcome variables are PRI, WLI, HR, and MBP. The occurrence of positive pain response after applying endotracheal intubation and surgical incision was reflected by hemodynamic reactivity, which 
was defined as more than $20 \%$ increase of either MBP or HR 1 minute after nociception stimulus [20, 21]. Receiver operating characteristics (ROC) curves and the associated areas under the curves (AUC) were computed to characterize the sensitivity and specificity and the ability of PRI and WLI (pre-stimulation values) in predicting hemodynamic reactivity. The asymptotic $95 \% \mathrm{Cl}$ of each AUC was calculated, as well as the asymptotic $P$ value under the null hypothesis that the true $A U C=0.5$. The reaction of $\mathrm{HR}$ and MBP after tracheal intubation and skin incision was compared by Wilcoxon signed-rank test.

Statistical analyses were performed using GraphPad Prism 5 (GraphPad Inc., USA), and MedCalc® version 12.1.4.0 (MedCalc Software, Mariakerke, Belgium). $P$ values less than 0.05 were considered significant.

\section{Results}

We initially recruited 163 pediatric patients during the study period, 13 patients were excluded because of fever and/or cough or refused to sign informed consent before surgery. 150 pediatric patients who met the inclusion criteria and obtained signed informed consent by their parents or legal guardians were included in this study. During the study, ten cases were excluded because of poor electrode-skin contact or noise interference, and another six cases were also excluded because of the use of atropine or vasoactive drugs, leaving 134 patients for final analysis (Figure 2). Patient characteristics are shown in Table 1. 114 children were males, and 20 were females. The median age of patients was 40 [IQR 17-64] months and the median weight was 15 [IQR 12-20] kg.

During induction of general anesthesia, the WLI values decreased much faster than the PRI values (Figure 1). The changes of HR, MBP induced by endotracheal intubation and surgical incision are respectively shown in Table 2. According to the criteria of hemodynamic reactivity, the positive reactivity of HR and MBP induced by endotracheal intubation was observed in 95 (70.9\%) and 61 (45.5\%) patients, respectively. Endotracheal intubation significantly increased the patient's HR and MBP $(P<0.001)$, the median values of HR and MBP changes were 30.4\% (IQR, 15.7-44.2\%) and 17.3 \% (IQR, 5.5-35.8\%), respectively; The positive reactivity of HR and MBP induced by skin incision was found in $19(14.2 \%)$ and $24(17.9 \%)$ patients. Skin incision significantly increased the patient's HR and MBP $(P<0.001)$, the median values of HR and MBP changes were 1.8\% (IQR, -2.7-9.9\%) and 7.1\% (IQR, 2.4-17.7\%), respectively.

To further analyze the abilities of PRI and WLI (pre-stimulation values) in predicting hemodynamic reactivity induced by endotracheal intubation and skin incision, receiver operating characteristics (ROCs) curves and the associated areas under the curves (AUCs) were computed (Table 3). Using either HR or MBP reactivity induced by tracheal intubation as a dichotomous variable, the AUCs [ $95 \% \mathrm{Cl}$ of $\mathrm{PRI}$ and WLI were 0.81 [0.73-0.87] and 0.58 [0.49-0.67], respectively, the best cutoff values (the optimal threshold) of PRI and WLI were 62 and 49 respectively; Using either HR or MBP reactivity induced by skin incision as a dichotomous variable, the AUCs [95\% Cl] of PRI and WLI were 0.82 [0.75-0.88] and 0.61 [0.52-0.69], respectively, the best cutoff values of PRI and WLI were 60 and 46, respectively. These results indicated 
that PRI was much better than WLI in predicting the hemodynamic reactivity induced by noxious stimulations during general anesthesia. The sensitivity and specificity of PRI and WLI with corresponding cutoff values in predicting hemodynamic reactivity were also included in Table 3. ROC curve of PRI and WLI to predict hemodynamic reactivity, HR change, and MBP change were depicted in Figure 3.

\section{Discussion}

In the present study, we present our preliminary validation study for PRI, a newly derived EEG parameter supposed to reflect antinociceptive state for surgical patients under general anesthesia. Using tracheal intubation and skin incision as noxious stimulation, we found that WLI cannot be used to predict the hemodynamic reactivity induced by tracheal intubation and skin incision despite being a similar parameter to BIS for monitoring the depth of sedation during general anesthesia; On the contrary, PRI can predict hemodynamic reactivity with the best cutoff values of 62 and 60 after tracheal intubation and skin incision in pediatric patients during general anesthesia. In this context, if the value of PRI is greater than 62 before intubation or 60 before skin incision, there was a high probability (AUC ROC $=0.81,0.82$ ) to find an increase of more than $20 \%$ in HR or MBP one minute after stimulation. The probability of PRI in predicting hemodynamic reactivity with corresponding cutoff values was higher than those of WLI. In addition, our study also demonstrated that WLI decreased significantly faster than PRI after induction of anesthesia, indicating WLI and PRI might reflect different characteristics of EEGs, EEGs from different brain regions or effects of different anesthetics. Further study is needed to confirm these results.

Self-report is considered as the gold standard in determining the presence and degree of pain perception in clinic practice, but it is not feasible for patients under general anesthesia, those suffering from consciousness disorders or are in coma. Therefore, more and more recent studies focus on methods of objective pain assessment. EEG-based pain assessment as a cheap, easy-to-use, and non-invasive technique has attracted a growing interest in recent years, and the positive relationship between EEG responses and pain perception has been relatively well established [22]. Pain index (PI), another EEG derived parameter used for pain assessment in awake patients from the same monitor (HXD-I multifunction combination monitor Beijing Easymonitor Technology Co., Ltd., China), was found to be significantly correlated with pain visual analogue scale/numerical rating scales [23]. But it cannot be used to assess the nociception of patients under general anesthesia, which might be due to the influence of general anesthetics on EEG [24].

General anesthesia significantly inhibits the activity of cerebral cortex, demonstrating that BIS is reduced, but leave the activity of autonomous nervous systems related sub cortexes relatively intact. Furthermore, noxious stimulations can still alter the activity of the cerebral cortex, demonstrating the hemodynamic changes. Therefore, several monitoring tools including ANI, Surgical Pleth Index (SPI), and Nociception level (NoL) have been developed to evaluate nociception during general anesthesia through the analysis of the relative parasympathetic tone. Researches have shown that these tools might reflect the nociception during general anesthesia $[1,16,21]$. However, these parameters are mainly aimed at adult patients and affected by arrhythmia alongside many heart rate affecting drugs [6, 25]. Pain Rating Index 
(PRI) is a cerebral pain tolerance indicator based on EEG signals. The nociception-related data in the whole EEG frequency band are then transformed by wavelet algorithm to obtain the PRI value (range 0100). Different from the rapid reduction of WLI, which represents the rapid hypnosis induced by propofol during anesthesia induction, the reduction of PRI is much slower, which might reflect combined effects of different brain regions activity and opioids. Furthermore, our study showed PRI values before noxious stimulation can be better to predict hemodynamic reactivity following noxious stimulations (intubation and skin incision) in children. Therefore, PRI might be clinically helpful to titrate the doses of opioids and predetermine whether an upcoming noxious stimulation would cause nociceptive response in pediatric patients.

Wavelet index (WLI) is a sedation indicator monitoring the depth of anesthesia developed by the same company $[18,19]$. In previous studies, BIS has been used to monitor the hypnotic component of anesthesia and was confirmed to be unable to predict hemodynamic changes $[5,26]$. In our study, WLI decreased significantly faster than the PRI which represents the rapid hypnosis, and also failed in predicting hemodynamic responses. This might be because that WLI and PRI reflect different characteristics and different brain regions of EEGs, or effects of different anesthetics.

In this study, we defined hemodynamic reactivity as an increase of more than $20 \%$ of either MBP or HR after 1 minute of painful stimulus. In adult patients, hemodynamic reactivity was often defined as an increase by more than $20 \%$ of HR and/or blood pressure within 5 min or following 2 min after noxious stimulation $[6,21,25]$. Through preliminary experiment, we found that in pediatric patients the hemodynamic response caused by nociception changes fast; Hemodynamic changes may have returned to normal five minutes after intubation or skin incision. In a study of children, MBP and HR values were recorded within one minute of the laryngeal mask airway insertion [20]. Therefore, we chose to the values of HR and MBP one minute after stimulation in order to detect hemodynamic reactivity caused by nociception more accurately.

Our study presents some limitations. First, some patients were excluded such as children with a history of epilepsy, autism, cognitive dysfunction. Patients receiving vasopressors, or ketamine before and during the experiment were also withdrawn. Therefore, our results may not be extrapolable to all patients. Second, our study was observational and anesthesia management including propofol and remifentanil dosing were left to the discretion of the anesthesiologist in charge, which might have induced bias in the analysis of the predictive ability. Third, our sample size was relatively small and the preliminary validation study was conducted in a single center. Larger sample and multi-center experiments are needed to be further carried out.

\section{Conclusion}

In conclusion, PRI can predict hemodynamic reactivity after tracheal intubation and skin incision in pediatric patients during general anesthesia. WLI, a similar parameter to BIS monitoring the depth of sedation, failed in predicting hemodynamic changes. This study suggests that PRI may be a reliable 
indicator of nociception during general anesthesia and may therefore constitute a useful analgesia monitor for children.

\section{Abbreviations}

ANI: Analgesia/Nociception Index; ASA: Anesthesiologists physical status; AUCs: Areas under the curves; BIS: Bispectral index; EEG: Electroencephalogram; PRI: Pain rating index; HR: Heart rate, HRV: Heart rate variability; IQR: Interquartile range; MBP: Mean blood pressure; NoL: Nociception level; ROC: Receiveroperating characteristic; SC: Skin conductance; SPI: Surgical Pleth Index; WLI: Wavelet index

\section{Declarations}

Acknowledgements: Not applicable.

Funding: This study was supported by the Medical Guide Project of the Shanghai Science and Technology Commission (16411967500) and the Shanghai Municipal Commission of Health and Family Planning, the Key Developing Disciplines (2015ZB0106). The funding bodies played the roles in the design of the study and collection, analysis, and interpretation of data.

Availability of data and materials: The datasets used and/or analyzed during the current study are available from the corresponding author.

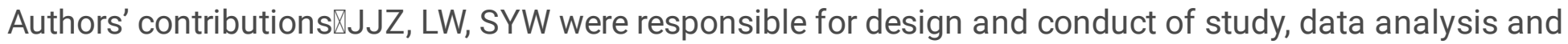
manuscript preparation. YTW, KZ were responsible for conduct of study, data analysis and manuscript preparation. JB, MZZ were responsible for data analysis and manuscript preparation.

\section{Ethics approval and consent to participate}

This study was approved by the Institutional Review Board of Shanghai Children's Medical Center (reference number: SCMCRB-k2018050, May 20, 2018) and registered in the www.chictr.org.cn (ChiCTR1800015969, May 3, 2018). The written informed consent was obtained from parents of the included pediatric patients.

Consent for publication: Informed consent was obtained and the consent form was signed by the parents.

Competing interests: The authors declare that they have no competing interests.

\section{References}

1. Edry R, Recea V, Dikust Y, Sessler DI. Preliminary Intraoperative Validation of the Nociception Level Index: A Noninvasive Nociception Monitor. Anesthesiology 2016, 125(1):193-203. 
2. Brown EN, Pavone KJ, Naranjo M. Multimodal General Anesthesia: Theory and Practice. Anesthesia and analgesia 2018.

3. Mashour GA. Neurophysiology and intraoperative nociception: new potentials? Anesthesiology 2013, 118(2):239-240.

4. Jensen EW, Valencia JF, Lopez A, Anglada T, Agusti M, Ramos Y, et al. Monitoring hypnotic effect and nociception with two EEG-derived indices, qCON and qNOX, during general anaesthesia. Acta anaesthesiologica Scandinavica 2014, 58(8):933-941.

5. Stockle PA, Julien M, Issa R, Decary E, Brulotte V, Drolet P, et al. Validation of the PMD100 and its NOL Index to detect nociception at different infusion regimen of remifentanil in patients under general anesthesia. Minerva anestesiologica 2018, 84(10):1160-1168.

6. Funcke S, Sauerlaender S, Pinnschmidt HO, Saugel B, Bremer K, Reuter DA, et al. Validation of Innovative Techniques for Monitoring Nociception during General Anesthesia: A Clinical Study Using Tetanic and Intracutaneous Electrical Stimulation. Anesthesiology 2017, 127(2):272-283.

7. von Dincklage F. Monitoring of pain, nociception, and analgesia under general anesthesia: Relevance, current scientific status, and clinical practice. Der Anaesthesist 2015, 64(10):758-764.

8. Malver LP, Brokjaer A, Staahl C, Graversen C, Andresen T, Drewes AM. Electroencephalography and analgesics. British journal of clinical pharmacology 2014, 77(1):72-95.

9. Guignard B. Monitoring analgesia. Best practice \& research Clinical anaesthesiology 2006, 20(1):161180.

10. Abad-Gurumeta A, Ripolles-Melchor J, Casans-Frances R, Calvo-Vecino JM. Monitoring of nociception, reality or fiction? Revista espanola de anestesiologia y reanimacion 2017, 64(7):406-414.

11. De Jonckheere J, Bonhomme V, Jeanne M, Boselli E, Gruenewald M, Logier R, et al. Physiological Signal Processing for Individualized Anti-nociception Management During General Anesthesia: a Review. Yearbook of medical informatics 2015, 10(1):95-101.

12. Mathews DM, Clark L, Johansen J, Matute E, Seshagiri CV. Increases in electroencephalogram and electromyogram variability are associated with an increased incidence of intraoperative somatic response. Anesthesia and analgesia 2012, 114(4):759-770.

13. Gjerstad AC, Storm H, Hagen R, Huiku M, Qvigstad E, Raeder J. Comparison of skin conductance with entropy during intubation, tetanic stimulation and emergence from general anaesthesia. Acta anaesthesiologica Scandinavica 2007, 51(1):8-15.

14. Sabourdin N, Barrois J, Louvet N, Rigouzzo A, Guye ML, Dadure C, et al. Pupillometry-guided Intraoperative Remifentanil Administration versus Standard Practice Influences Opioid Use: A 
Randomized Study. Anesthesiology 2017, 127(2):284-292.

15. Okkesim S, Celik G, Yildirim MS, Ilhan MM, Karaman O, Tasan E, et al. Comparison of Pulse Rate Variability and Heart Rate Variability for Hypoglycemia Syndrome. Methods of information in medicine 2016, 55(3):250-257.

16. Won YJ, Lim BG, Lee SH, Park S, Kim H, Lee IO, et al. Comparison of relative oxycodone consumption in surgical pleth index-guided analgesia versus conventional analgesia during sevoflurane anesthesia: A randomized controlled trial. Medicine 2016, 95(35):e4743.

17. Shoushtarian M, Sahinovic MM, Absalom AR, Kalmar AF, Vereecke HE, Liley DT, et al. Comparisons of Electroencephalographically Derived Measures of Hypnosis and Antinociception in Response to Standardized Stimuli During Target-Controlled Propofol-Remifentanil Anesthesia. Anesthesia and analgesia 2016, 122(2):382-392.

18. Yang N, Ge MF, Wang TL, Wu XG. Feasibility analysis of wavelet index for monitoring the depth of anesthesia in patients undergoing general anesthesia. Zhonghua yi xue za zhi 2011, 91(40):2849-2852.

19. Zhang XT, Cheng H, Xiong W, Wang BG. Comparison of the ability of wavelet index and bispectral index for reflecting regain of consciousness in patients undergone surgery. Chinese medical journal 2010, 123(12):1520-1523.

20. Kim HS, Park HJ, Kim CS, Lee JR. Combination of propofol and remifentanil target-controlled infusion for laryngeal mask airway insertion in children. Minerva anestesiologica 2011, 77(7):687-692.

21. Boselli E, Bouvet L, Begou G, Torkmani S, Allaouchiche B. Prediction of hemodynamic reactivity during total intravenous anesthesia for suspension laryngoscopy using Analgesia/Nociception Index (ANI): a prospective observational study. Minerva anestesiologica 2015, 81(3):288-297.

22. Bai Y, Huang G, Tu Y, Tan A, Hung YS, Zhang Z. Normalization of Pain-Evoked Neural Responses Using Spontaneous EEG Improves the Performance of EEG-Based Cross-Individual Pain Prediction. Frontiers in computational neuroscience 2016, 10:31.

23. An JX, Wang Y, Cope DK, Williams JP. Quantitative Evaluation of Pain with Pain Index Extracted from Electroencephalogram. Chinese medical journal 2017, 130(16):1926-1931.

24. Sahinovic MM, Eleveld DJ, Kalmar AF, Heeremans EH, De Smet T, Seshagiri CV, et al. Accuracy of the composite variability index as a measure of the balance between nociception and antinociception during anesthesia. Anesthesia and analgesia 2014, 119(2):288-301.

25. Boselli E, Logier R, Bouvet $L$, Allaouchiche $B$. Prediction of hemodynamic reactivity using dynamic variations of Analgesia/Nociception Index (ANI). J Clin Monit Comput 2016, 30(6):977-984. 
26. Khandelwal P, Gombar KK, Ahuja V, Gombar S. Tracheal intubation with volatile induction and target bispectral index of 25 versus 40: A randomized clinical trial. Journal of anaesthesiology, clinical pharmacology 2016, 32(3):349-352.

\section{Table}

Table 1 Patient Characteristics $\square$ N. $=134 \square$

\begin{tabular}{ll}
\hline Patient Characteristics & Values \\
\hline Age (months) & $40(17,64)$ \\
Gender & \\
$\quad$ Male & 114 \\
$\quad$ Female & 20 \\
Height, cm & $99 \pm 20$ \\
Weight, kg & $15(12,20)$ \\
BMI, kg/m ${ }^{2}$ & $16(15,17)$ \\
\hline
\end{tabular}

Data are expressed as mean \pm SD or median with interquartile range.

$\mathrm{BMI}=$ body mass index

Table 2 Prestimulus, Poststimulus (Median [25-75th Percentile]) of MBP, HR after intubation and skin incision

\begin{tabular}{lll}
\hline & MBP & HR \\
\cline { 2 - 3 } & Median (25-75\%) & Median (25-75\%) \\
\hline TP1 pre & $65(59.9$ to 74$)$ & $91.5(81$ to 104.3$)$ \\
TP1 post & $78(66.3$ to 92.8$)$ & $124.5(108.5$ to 135$)$ \\
TP1 reaction & $17.3 \%(5.5$ to $35.8 \%)$ & $30.4 \%(15.7$ to $44.2 \%)$ \\
P value & $<0.001$ & $<0.001$ \\
TP2 pre & $61.7(55.3$ to 67$)$ & $94.5(81$ to 109$)$ \\
TP2 post & $67.2(59.3$ to 75$)$ & $100.5(80.8$ to 118$)$ \\
TP2 reaction & $7.1 \%(2.4$ to $17.7 \%)$ & $1.8 \%(-2.7$ to $9.9 \%)$ \\
P value & $<0.001$ & $<0.001$ \\
\hline
\end{tabular}

Reaction $\mathrm{i}=$; $\mathrm{HR}=$ heart rate; $\mathrm{MBP}=$ mean blood pressure; $\mathrm{TP} 1=$ tracheal intubation; $\mathrm{TP} 2=$ skin incision; post $=$ poststimulus; pre $=$ prestimulus 
Table 3 Prognostic Power of PRI and WLI (pre-stimulation values) in Predicting Hemodynamic Response

\begin{tabular}{|c|c|c|c|c|c|c|}
\hline & & Threshold & Sensitivity, \% & Specificity, \% & AUC (95\%CI) & $\mathrm{P}$ \\
\hline \multirow[t]{6}{*}{ PRI } & MBP (TP1) & $>60$ & 85.2 & 49.3 & $0.68(0.59-0.76)$ & $<0.01$ \\
\hline & HR (TP1) & $>58$ & 88.4 & 66.7 & $0.81(0.74-0.88)$ & $<0.01$ \\
\hline & MBP or HR (TP1) & $>62$ & 80.8 & 71.4 & $0.81(0.73-0.87)$ & $<0.01$ \\
\hline & MBP (TP2) & $>57$ & 79.1 & 81.8 & $0.79(0.71-0.85)$ & $<0.01$ \\
\hline & HR (TP2) & $>58$ & 73.7 & 80.9 & $0.77(0.68-0.83)$ & $<0.01$ \\
\hline & MBP or HR (TP2) & $>60$ & 78.1 & 86.3 & $0.82(0.75-0.88)$ & $<0.01$ \\
\hline \multirow[t]{6}{*}{ WLI } & MBP (TP1) & $>44$ & 77.1 & 49.3 & $0.61(0.52-0.69)$ & 0.03 \\
\hline & HR (TP1) & $>43$ & 70.5 & 35.9 & $0.51(0.42-0.60)$ & 0.84 \\
\hline & MBP or HR (TP1) & $>49$ & 52.5 & 68.6 & $0.58(0.49-0.67)$ & 0.14 \\
\hline & MBP (TP2) & $>47$ & 66.7 & 55.5 & $0.62(0.53-0.70)$ & 0.08 \\
\hline & HR (TP2) & $>46$ & 68.4 & 49.6 & $0.55(0.47-0.64)$ & 0.38 \\
\hline & MBP or HR (TP2) & $>46$ & 68.8 & 51.9 & $0.61(0.52-0.69)$ & 0.06 \\
\hline
\end{tabular}

The asymptotic $\mathrm{P}$ value under the null hypothesis that the true AUC $=0.5 ; \mathrm{PRI}=$ pain rating index; $\mathrm{WLI}=$ wavelet index; $\mathrm{HR}=$ heart rate; $\mathrm{MBP}=$ mean blood pressure; $\mathrm{TP} 1=$ tracheal intubation; $\mathrm{TP} 2$ = skin incision; post = poststimulus; pre $=$ prestimulus

\section{Figures}




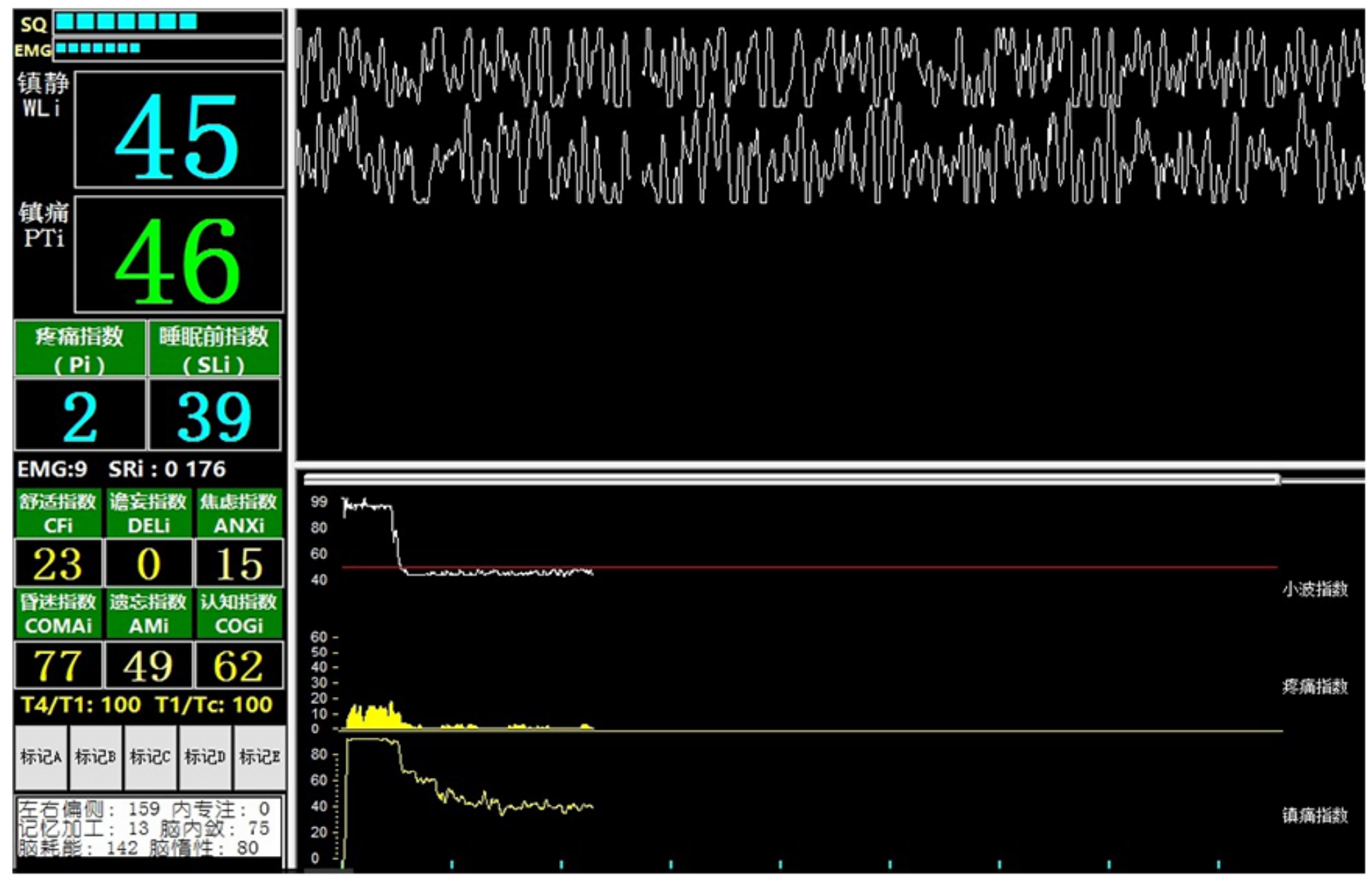

\section{Figure 1}

Real-time data of the monitoring devices White line= real-time data of WLI; yellow line= real-time data of PRI 


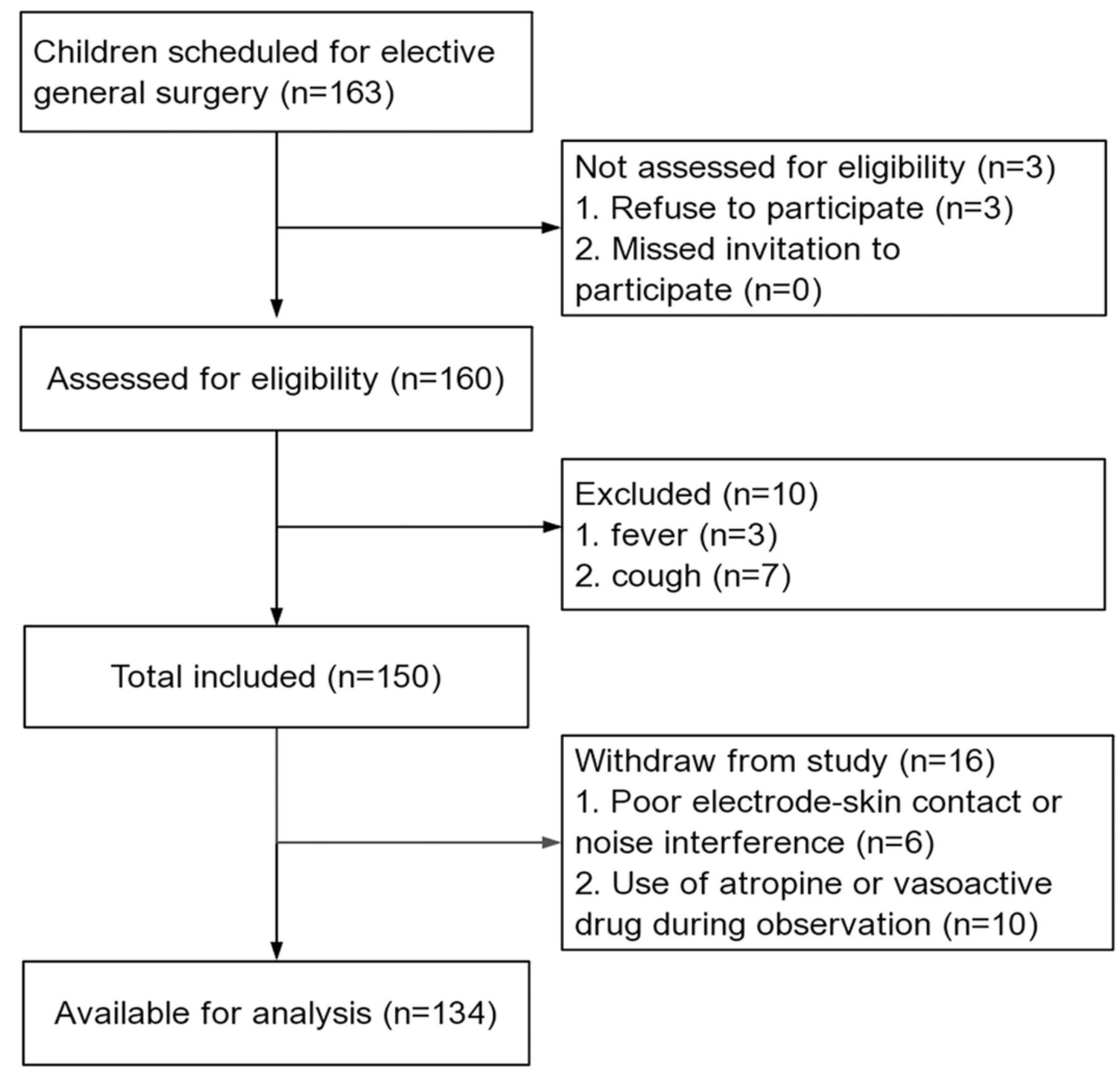

Figure 2

The CONSORT flow diagram 

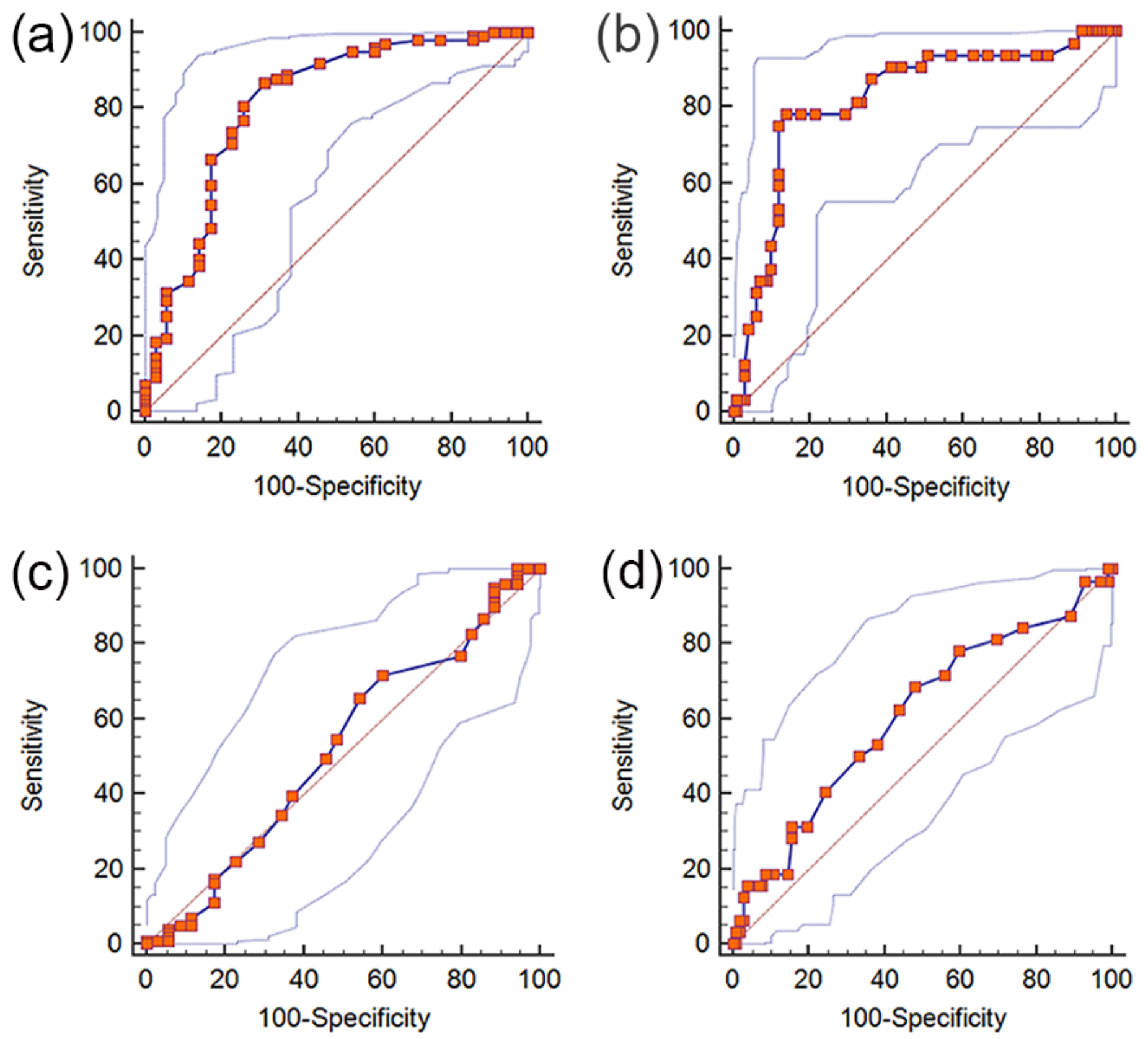

Figure 3

ROC curves of PRI and WLI for the prediction of hemodynamic reactivity ROC curves of PRI for the prediction of hemodynamic reactivity after tracheal intubation (a) and skin incision (b); ROC curves of WLI for the prediction of hemodynamic reactivity after tracheal intubation (c) and skin incision (d) 